\title{
Insuficiencia Renal Crónica (I.R.C.)
}

La I.R.C. afecta funcionalmente no sólo a las sustancias que se excretan por la orina, sino también a la regulación del medio interno.

Muchas veces debemos conformarnos con c) diagnóstico global de Insuficiencia renal sin poder determinar la enfermedad de base y esto es por la complejidad de las lesiones que hace que aun el anatomopatólogo tenga dificultades en comprobar la naturaleza de la afección primaria.

Aunque toda falla prolongada de una o varias funciones del rî́ńon pueden considerarse como IRC, como ocurre con los síndromes nefróticos, tubulopatías, etc., al mencionar este diagnóstico nuestra atención se ditige a Ias enfermedades que se manifiestan por el aumento de la uremia, con o sin hipertensión, es decir, a la caída de la función glomerular y del nefrón en general.

Podemos definir a la insuficiencia renal c1ónica como la ređucción de más de un $50 \%$ d: la filtración glomerular en forma mantenida e irrecuperable (Nephron 7; 385; 1970). - bien como una destrucción irreversible y en general progresiva de los nefrones, de lo que resulta un trastorno del medio interno (Falbriand y Engal. Ciba 1964).

Esta disminución global y progresiva de

\footnotetext{
tDepartamento Nefrourología, Hospital Luis Calvo Mackenna.
}

Drs. C. Saich A.*, F. Puga C.*, J. Weinberger*.

1) función renal es causada por la extension paulatina del daño histológico o su evolución hacia la esclerosis que afecta a los glomérulos. a los túbulos, al intersticio y a los vasos, aunque casi siempre encontranios un predoninio inicial de algunas de estas estructuras. La etiología putede ser múltipie, variando el mecanismo patogénico. Tambiên puede ser diferente el comienzo de la lesión, pero cuancio la nefropatía llega a la etapa terminal, el síndrome clínico - humoral - metabólico - urinario e histológico es casi idéntico para todos ellos.

No existe, en la mayoría de Jos casos paralelismo entre la histología, radiología y sedimento urinario alterado, con el grado de perturbación de la función renal.

Se acepta que la función renal en la IRC está mantenida por la persistencia de nefrones indemnes (Teoría de los nefrones intactos) y no por daños segmentarios de éstos.

Por consiguiente, el progreso hacia la IRC está condicionado por el compromiso creciente de nefrones completos, lo que explica las rtapas de poliuria con hipostenuria (incapacidad de concentración), poliuria con isostenuria (nćrdida de la capacidad de dilución) y la olietria con isostentiria (pérdida de la capacidad de concentración y dilución).

Como se dijo antes. las causas que producen IRC en la infancia son múltiples; una clasificación se propone en Ta Tabla $N$ ․ 1 . 

a) Glomerulares:
- Primarias
- Hereditarias
(Hialinosis)
- Secundarias
(Alport)
- Infecciones
(Sch. Henoch)
b) Túbulo-Intersticiales:
- Metabólicas
(Pielonefritis)
- Otras
(Nefrocalcinosis)
(Drogas)
c) Congénitas:
- Riñón qústico
- Displasía renal
d) Vasculares:
e) Tumores:

En el Hospital Luis Calvo Mackenna, los niños con diagnóstico de IRC en los últimos 10 años comprenden el $0.08 \%$ del total de hospitalizaciones (99 niños), de los cuales hasta el momento han fallecido el 44,4\% (44 niños).

En la Tabla No 2 se detallan desde el punto de vista etiológico las causas de IRC en 99 niños hospitalizados y faltecidos entre los años 1963 y 1972.

$$
\text { Tabla V? } 2
$$

CAUSAS DE I.R.C. EN NINOS HOSPITALIZADOS Y FALLECIDOS

"HOSPITAL LUIS CALVO MACKENNA"

$$
1963-1972
$$

\begin{tabular}{lccc}
\hline \multicolumn{1}{c}{ Catusa } & Hosp. & Fallecidos & $\%$ \\
\hline Pielonefritis crónica & 43 & 19 & 43.15 \\
Glondérulonefritis cr. & 32 & 14 & 31.80 \\
L.E.D. & 5 & 4 & 9.05 \\
Schönlein Henoch & 5 & 2 & 4.50 \\
S. hemolitico urémico & 4 & - & - \\
Amiloidosis & 2 & 1 & 2.30 \\
Rińón quístico & 2 & 1 & 2.30 \\
Nefronoptisis & 2 & 1 & 2.30 \\
Hipoplasia renal & 2 & 1 & 2.30 \\
Nefroesclerosis & 1 & - & - \\
Nefritis uricémica & 1 & & $100 \%$ \\
\end{tabular}


Como casi todas las enfermedades crónicas, que a la larga llevan a la muerie, los niños con IRC consultan tardíamente. Es excepcional que un paciente sea observado progresar hacia este desastre y en cambio muy corriente que su primera consulta se lleve a efecto poco tiempo antes de morir, habiendo sido aparentemente sano hasta ese momento. Los antecedenles son también pobres, muy rara vez hay nefropatia en los parientes.

Podemos distinguir 3 etapas que van a estar dadas fundamentalmente por la extensión de la destrucción de las nefronas:

\section{A.- INSUFICIENCIA RENAL LATENTE}

Cincuenta por ciento de destrucción de las nefronas. El riñón es suficiente para las exigencias de vida corriente y no hay manifestaciones clínicas, ni humorales; sólo alteraciones urinarias.

\section{B.- INSUFICIENCIA FENAL COMPENSADA}

Entre el $50 \%$ y $70 \%$ de nefronas destruidas. Estos pacientes tienen elementos clínicos y urinatios.

\section{C - INSUFICIENCIA RENAL DESCOMPENSADA}

La destrtcción sobrepasa el $70 \%$. Se manifiesta claramente el síndrome clínico-urinario liumoral.

\section{CUADRO CLINICO}

El primer indicio puede ser la fatígabilidad, palidez, anorexia progresiva y fiebre ocasional. Sed variable con frecuentes levantadas en la noche a tomar agua y orinar. En los nin̄os con IRC suele interrumpirse el crecimiento antes que aparezca el síndrome clínico.

La hipertensión es uno de los trastornos más importantes vinculados con un IRC. Sin embargo, suele estar ausente. Puede deberse a ruecanismo tipo Goldblatt, por participación suprarrenal y del aparato yuxta-glomerular o en la etapa terminal, a la retención de sodio y daño vascular. Si la hipertensión es mantenida se apreciarán todas las repercusiones cardiovasculares conocidas.

No es rara la aparición de derrames pleurales en el síndrome ureico o simplemente edema pulmonar, cuyo diagnóstico es radiológico. La disnea y los trastornos del titmo son frecuentes y están vinculados a las alteraciones del pH, los desórdenes hidroelectrolificos o insuficiencia cardíaca.

Con frecuencia se produce una pericarditis si el BUN permanece alto $y$ constituye un signo desfavorable.

La insuficiencia cardíaca responde a una combinación de anemia, hipertensión y los trastornos bioquímicos generales propios del síndrome urémico. A menudo es la complicación terminal.

Anorexia, náuseas y vómitos son la regla, ocasionando adelgazamiento y desbidratación, lo que empeora la función renal por hipoperfusión. La diarrea es frecuente, y por fragilidad capilar y ulceraciones locales se presentan hemorragias digestivas; estos trastornos son debido a la eliminación de urea y otros catabolitos proteicos por las mucosas digestivas.

Desde el punto de vista digestivo encontramos estomatitis, aliento fétido, derivado de la eliminación de urea en la saliva y su transformación amonfacal con aparición de gérmenes, contribuyendo al desarrollo de la estomatitis fetida y sangrante.

Las thanifestaciones del síndrome neuromuscular pueden ser centrales o periféricas, las centrales son mareos, somnolencia y coma.

Las periféricas se caracterizan por parestєsias, dolores y calambres musculares, acom̧̧añados a veces de fibrilaciones.

Las causas de estas molestias no son del todo claras, pero se invocan la hipocalcemia, hiperfosfemia, hipermagnasemia, acidosis $y$ alteraciones enzimáticas celulares.

El síndrome hematológico está caracterizado por perturbaciones en los glóbulos rojos, anenia por déficit de eritropoyetina y dismi- 
nución de su vida media. Son frecuentes las perturbaciones de la hemostasis con disminución de los elementos del complcjo protrombinico, alteraciones de las plaquetas y una disminución de la resistencia capilar.

Dentro de las manifestaciones clérmicas es habitual el tinte amarillo de la piel, debido a la fijación del urocromógeno. Hay sequedad y prurito, siendo la dermatitis mal definida, apareciendo a veces las lesiones denominadas urémides, que revelan la eliminación de urea por la piel, semejante a trozos de escarcha.

El compromiso del sistema óseo aparece tardíamente en los niños y se caracteriza por dolores y deformación. Radiológicanente aparece el esqueleto descalcificado (ostcodistrofia tenal).

Cuando se presenta en época de gran crecimiento puede dar lugar a un enanismo o raquitismo renal. Secundariamente puede haber manifestaciones endocrinas caracterizadas por un hiperparatiroidismo.

\section{TRATAMIENTO}

Los recursos para el tratamiento de estos Los recursos para el tratamiento de estos pacientes son extraordinariamente limitados, dado lo avanzado de la enfermedad cuando ellos son pesquisados. Tal vez una intervención quirúrgica para liberar una uropatía obstructiva podtía prolongar la vida de ese niño. No se obtienen resultados favorables en la curación de un reflujo o de una obstrucción postvesical cuando ya el daño renal se ha establecido.

Sin embargo, hay medidas paliativas que hacen más soportables la vida y aun podrían prolongarla.

Fundamental es considerar los aspectos psicológicos del niño y su familia, que adquieren mayor importancia a medida que cl niño empeora.

En las fases iniciales no hace falta ringuna dieta especial. El agua debe administrarse "ad libitum" hasta que se llega a oliguria con isostenuria.

Las restricciones de sodio se hatán solamente en los casos de hipertensión severa, y si ésta no existe, adecuarlas de acterdo con las cantidades de sodio eliminadas por la orina cn 24 horas.

Las proteínas deben limitarse sólo cuando cl nivel de uremia comienza a ascender, buscando un balance adecuado, pero preocupándose especialmente en el aporte total de calorías, especialmente en base a bidrato de carbono.

La hipocalcemia y el fósforo inorgánico elevados no son susceptibles de modificarlos significativamente con medidas dietéticas. sunque se puede limitar alimentos con gran contenido en fósforo, como la leche y administrar gel de hidróxido de aluminio para disminuir la absorción de fósforo y aumentado la ingestión de calcio.

Si existe hipocalcemia marcada se debc administrar sales de calcio, vitamina D (1-25 cilhidro colecalciferol) y prevenir la osteodistrofia. El uso de vitamina D debe ser controlado, ya que ésta se acumula y puede aparecet hipercalcemia. Una acidosis leve 0 moderada no requiere tratamiento y solamente en caso de reserva alcalina baja daremos bicarbonato sódico "per os", no intentando corregir total ni bruscamente la acidosis por cl peligro de tetania o convulsiones.

Los diuréticos sólo deben ser usados en caso de edema o bipertensión, cuidando la concentración de iones en el plasma. La hipertensión se tratará cuando es sintomática a sobrepasa los $90 \mathrm{~mm} \mathrm{Hg}$ la diastólica, siempre considerando que los hipotensores deprimen la función renal.

La transfusión debe indicarse cuando se considera que la anemia contribuye a agravar la sintomatología del niño y teniendo en consideración que cada transfusión aporta una mayor cantidad de antígenos, lo que ateja al paciente de la posibilidad de traspiante.

El resto de las complicaciones recibe tratámiento sintomático tratando de llevar al paciente a las mejores condiciones.

En relación a la actividad, debe permitírseles escoget el ritmo de vida, $\sin$ deportes competitivos. La asistencia a la escuela debe ser regular mientras la enfermedad se lo permita. 
Cuando la función renal está muy disminuida, sólo queda como recurso la dialisis crónica, la que en el niño es una etapa hacia el trasplante renal.

\section{RESUMEN}

Se analiza la etiología de la insuficiencia renal crónica esbozando una clasificación de las causas que la producen, sus manifestaciones clínicas más frecuentes $\mathrm{y}$ su tratamiento actual.

Se esiudian además 99 niños hospitalizados entre los años 1963 y 1972 con diagnóstico de insuficiencia renal crónica en el Hospital Lutis Calvo Mackenna, examinando la incidencia, la etiología, edad de comienzo y la mortalidad.

\section{SUMMARY}

The etiology of chronic renal failure is snalized through a clasification of its most frequent causes, its clinical presentation and the actual recommended management.

Ninety nine children admited in the Luis Calvo Mackenna Children's Hospital from 1963 to 1972 with the diagnostic of chronic renal failure are analized with special emphasise of the incidence, etiology, age of presentation and mortality founded in these case.

\section{REFERENCIAS}

1. M.S.F. Mc Lachlan et at.: Arch. of Dis, in Child. 1975. 50: 253.

2. S.M. Baileg et al.: The Lancet. 1975, 12 July 57; Vol. 2.

3. J.Y. Gillenmeter: J. Urol., 1971. t06: 122.

4. Nephron. 1970. 7; 385.

5. Insuf. Renal Cronical. Ciba, 1974.

6. F. Puga y col.: Rev. Chil. Ped. 1974. 44: 557.

7. J. James: Renal Diseases in Childhood. The C. V. Mosby Company, 1970.

8. F. Puga; Nefrologín. Ed. A. Bello, 1968.

9. Bricker, N.S. et al.: Am. J. Med. 1960, 28: 77. 